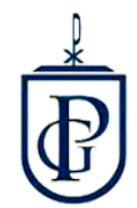

Veritas Et Scientia

Vol. $7, N^{\circ} 1,816-821$

Enero - Junio del 2018.

ISSN $2307-5139$

\title{
LA DESPENALIZACIÓN DE LA MARIHUANA PARA "USO TERAPÉUTICO" Y LA ECONOMÍA SOCIAL DE MERCADO EN TACNA AÑO 2015
}

\author{
HE DEPENALIZATION OF MARIJUANA FOR "THERAPEUTIC USE" AND THE SOCIAL MARKET ECONOMY IN TACNA, \\ YEAR 2015
}

Wilfredo Hilario Caballero Laura ${ }^{1}$

\section{RESUMEN}

El presente trabajo trata de "La Despenalización de la Marihuana para uso terapéutico y la economía social de mercado en Tacna año 2015”. Los objetivos de la presente investigación, conducen a la seguridad jurídica de la despenalización de la marihuana para "uso terapéutico" y la Economía Social de Mercado. Y dentro de ello, los objetivos específicos como son; los aspectos Legislativo, Judicial y Politico. Investigación aplicada, que se dirige a la solución del problema. Para tal efecto se utilizó la información obtenida a través del cuestionario y la entrevista. Se ha llegado a concluir que los aspectos; Legislativo, Judicial, y Político de la Despenalización de la Marihuana para "uso terapéutico", influyen positivamente en la Economía Social de Mercado.

Palabras claves: Drogas sociales, Marihuana, Despenalizar Economía Social de Mercado.

\section{ABSTRACT}

The present work deals with "The Decriminalization of Marijuana for therapeutic use and the social market economy in Tacna 2015". The objectives of the present investigation, lead to the legal security of the decriminalization of marijuana for "therapeutic use" and the Social Market Economy. And within it, the specific objectives as they are; the Legislative, Judicial and Political aspects. Applied research, which is aimed at solving the problem. For this purpose, the information obtained through the questionnaire and the interview was used. It has come to conclude that the aspects; Legislative, Judicial, and Political of the Decriminalization of Marijuana for "therapeutic use", positively influence the Social Market Economy.

Keywords: Social Drugs, Marijuana, Decriminalize Social Market Economy

\footnotetext{
${ }^{1}$ Maestro en Derecho Constitucional
} 


\section{INTRODUCCIÓN}

El propósito de la presente investigación pretende proponer la despenalización de la marihuana (cannabis sativa), para "uso terapéutico", basicamente invocando el derecho Constitucional a la salud que ostenta el ser humano por ser tal, y como demanda social. Estos derechos están reconocidos en diversos Tratados Internacionales, en los que el Peru es parte. Aquí, se precisa desarrollar la distinción entre el uso y el abuso de la marihuana, considerando al primero como una opcion estimada positivamente, en la medida que su destino sea solo para "uso terapéutico". La marihuana se distingue de otras drogas, cuyas propiedades curativas ha logrado resultados espectaculares en tratamientos de diversos males tales como; el cáncer, esclerosis, glaucoma, migrañas, enfermedades crónicas, diabetes, alzheimer, convulsiones, nauseas, etcétera. Sin embargo, hay que lamentar el poco interés político mostrado en el contexto nacional, que deriva en el desperdicio de esta polemizada planta, cuyos pretextos principales son el crecimiento del narcotráfico y la dependencia al consumo adictivo. Estas realidades son complejas y se sustentan en vacíos legales de la legislación nacional, es innegable que estos presupuestos impulsan el mercado negro. La política represiva del Estado, penaría en todas sus formas el manejo de la marihuana. En principio, solo si existiera colisión con el bien jurídico protegido, principalmente la salud pública. La finalidad de esta investigación propone atender un problema de salud latente, porque los efectos de la misma, buscan la despenalización y regulación de la marihuana para "uso terapéutico", descartando de plano el uso recreativo de la droga.

Pesan dos contrariedades gravitantes que influyen de manera negativa en la concreción de la presente propuesta; el narcotráfico, y las secuelas inmediatas que afectan la Economía Social de Mercado. Se criminaliza de manera constante a los usuarios de la marihuana, no hay razón, más aún si este consumo está destinado para fines medicinales. Las políticas actuales de gobierno afectan a toda la sociedad, y no soluciona el problema, pues la demanda de drogas, detona la actividad ilícita de grupos criminales sin importarles el costo social que trae consigo. La otra demanda refiere en términos de salud, que viene siendo entorpecida por nuestra Legislación actual, la Ley Penal atenta contra el derecho y la libertad de la persona para tomar sus propias decisiones. No tenemos un Derecho Penal alternativo, en el que las garantías vigentes transmitan al proceso penal, la promoción y la consecución de la paz social. No es difícil constatar que la represividad del tráfico de drogas no ha disminuido el negocio ilícito ni su consumo, en materia de salud no hay políticas sanitarias efectivas. Finalmente hay que aceptar que políticamente el crimen organizado asociado al tráfico de drogas, deviene en violencia y corrupción, este flagelo es uno de las causas principales que atentan el desarrollo de la economía del Pais.

Perez y Merino (2012), afirman que las enfermedades mentales o psicosis, muchas veces son predispuestas para la comisión de los delitos, aquí se considera el delito como una enfermedad, porque las características son mentales y psicopatológicas. Calvo (2016), considera al decomiso como la confiscación a los instrumentos materia del delito, la condena incumbe la pérdida de ellos. Marzal (1998), afirma respecto a etnología criminal, que son costumbres de la convivencia humana y de las reglas jurídicas de los pueblos primitivos de nuestras sociedades. Esta forma es parte de la etnología jurídica. Allport (1985), menciona que la personalidad es la muestra de la conducta que incide en el comportamiento de un individuo, es decir, de los sistemas psicofísicos que determina una forma de pensar y de actuar, única en cada sujeto en su proceso de adaptación al medio, en el que podemos descartar los rasgos particulares, y que mas bien esta es producto de su integración social y cultural. La Organización mundial de la Salud desde 1969, afirma que la rehabilitación es parte de la asistencia médica encargada de desarrollar las capacidades funcionales y psicológicas del individuo, a fin de permitirle llevar una existencia autónoma y dinámica. Es la capacidad de Restituir o habilitar nuevamente a una persona o cosa a su antigua situación jurídica. 
Fernandez y Espejo (2002), consideran a la marihuana, sustancia natural o sintética que produce adicción, esta planta procede del cáñamo índico (cannabis sativa), La resina llamada hachís es mucho más potente que las hojas o la flor. Es así que el fumador de marihuana adopta actitudes pasivas, inhibiéndose del mundo real pero que luego adopta actitudes emocionales. La OMS. (1948), refiere que droga es cualquier sustancia que puede pejudicar al cuerpo humano al ser ingerida por cualquier medio, alterando el sistema nervioso central del individuo, esta puede crear dependencia de tipo físico y psicologico. Kramer y Cameron (1975) arrimaban el concepto de droga como toda sustancia que introducida en el organismo vivo, puede modificar una o más funciones de este. Pero esta aclaración no es del todo clara y contundente, es decir, distinguir si la farmacodependencia es una sustancia capaz de producir peligro en la humanidad de la persona, no se llega a inferir de forma precisa este concepto. Dugdale (2012), afirma que la fármacodependencia es el estado psíquico y físico del individuo, producido por la ingesta de fármacos, la particularidad del caso es la modificacion del comportamiento que comprenden un impulso irreprimible de tomar un fármaco en forma continua para experimentar sus efectos psíquicos causados siempre por la privación del fármaco.

Bouquet (1950, p. 97), afirma que la marihuana "Es originaria de Asia Central, pero también crece en regiones templadas, asi como en las tropicales, mayormente esta dirigida al comercio. La marihuana es utilizada como insumo para producir fibras (cáñamo), además del aprovechamiento de la sustancia psicoactiva presentes en sus hojas y sumidades de la planta. Puede llegar a crecer desde 1.50 $\mathrm{m}$., hasta los $6.00 \mathrm{~m}$. de altura, dependiendo de las condiciones de tierra y clima."

Los constituyentes químicos de la marihuana "...son muy complejos y comprenden un grupo singular de sustancias químicas denominadas cannabinoides" Se pueden dar cuenta de cinco cannabinoides entre los más importantes, conforme a la actividad biológica de la planta (Mechoulam, 1970, p. 79). El cultivo de cannabis depende mucho de diversas acepciones, y una de ellas es el suelo y clima, es así que, según las muestras de semillas originarias de Canada, los Estados Unidos de América y Europa Occidental, se consiguió un cannabis con escaso contenido de Delta 9- THC., pero de buen promedio de la sustancia cannabidiol. (Fetterman, 1970). Por otra parte, de las semillas originarias de México y Tailandia; se consiguió preparado con alto contenido de Delta 9-THC. Pero con respecto al cannabis cultivado en los Estados Unidos de América, estas contienen apenas 0,09\% de Delta 9-THC, contrariamente los que procedían de Tailandia registraban 4,89\%. (Turk, 1970).

En Holanda, acuerdan que la política trazada en materia de drogas de este país, si distingue las drogas duras de las drogas blandas, es decir las anfetaminas, la cocaína, la heroína y otras sustancias son consideradas peligrosas. Por el contrario, la marihuana, el hachís, los derivados del cáñamocannabis sativa, ademas del nederwiet (cultivo holandés de marihuana), son aprovechadas para el uso recreativo y medicinal, este consumo esta despenalizado si la persona posee una dosis personal autorizado por el legislador. En 1976 se llega a descriminalizar la marihuana. Se había logrado el consumo libre del cannabis, pero a cuenta de que estos se destinasen para usos medicinales y de recreación, el máximo permitido era de 30 gramos, la tenencia de más de 30 gramos constituia un delito penal. Los cafés (coffeeshops) holandeses, están habilitados para los usuarios de cannabis. (Blickman y Jelsma, 2012). Canada viene estudiando la manera de impulsar, la medicina terapéutica del cannabis en beneficio propio. Es asi que, en el 2004, desplegó el último proyecto de ley con la finalidad establecer cambios en la ley de drogas actual. En dicho proyecto se pretendió despenalizar la tenencia de cantidades minimas de marihuana, solo hasta 15 gramos para uso individual. Así, la Corte Suprema de Canadá con plena autonomía, confirmó el mandato constitucional del Parlamento canadiense con la finalidad de vedar la tenencia de marihuana, mediante su sistema normativo penal en el 2003.

En Uruguay, en 1998 se establecieron las modificaciones a la Ley de Estupefacientes Na 17.016; referente a estupefacientes y sustancias que determinen dependencia física o psíquica, el artículo 31 párrafo segundo dice: "Quedará exento de pena el que tuviere en su poder una cantidad razonable destinada exclusivamente a su consumo personal con arreglo a la convicción moral que se forme el 
juez a su respecto, debiendo fundamentar en su fallo las razones que la han formado" Garat (2011). Posteriormente, se presentaron varios proyectos de ley para modificar la legislación actual con respecto a la marihuana permitiendo el auto cultivo. En el 2012 se planteó desde el Poder Ejecutivo la posibilidad de aprobar un proyecto de ley, para que el Estado se haga cargo de la producción y comercialización de esta sustancia. Así, el 4 de marzo de 2012, se dispone el proyecto de ley que legaliza la venta de marihuana, en el que se entregaran entre 20 y 40 licencias a personas privadas para su producción, estas tendrían un costo y se entregarán a personas con capacidad moral. La fecha histórica para Uruguay es el 10 de diciembre de 2013, este pais se convirtió en el primer país del mundo en legalizar la producción, tenencia y uso de marihuana, logrando la legalidad completa de este producto. La marihuana se venderá en farmacias a mayores de 18 años de edad, pudiendo adquirirse hasta 40 gramos por persona. En un debate legislativo con 16 votos a favor, el Senado ha aprobado la iniciativa promovida por el ex presidente José Mujica el 2012, con el propósito de combatir al narcotráfico. (Garat, 2011). No hay que olvidar, que se debe admitir un conjunto de argumentos basados en principios filosóficos, científicos, políticos e históricos en pro de la legalización. (Doumet, 1992)

Peyraube y Bouso (2015) afirman; que la marihuana (cannabis sativa), se ha manipulado como medicina desde hace milenios por diversas culturas en determinadas partes del mundo. Además, se han descubierto muchas propiedades medicinales provenientes de otros cannabinoides. Los terpenos y los flavonoides de la planta, resultaron también tener propiedades terapéuticas.

Debemos saber diferenciar lo que es producción y tráfico. Alessandro Baratta (1993) estima: "Como si por el simple hecho de no utilizar o utilizar menos el instrumento penal para el control, la producción, el tráfico y el suministro de drogas debieran quedar sin ningún control, tanto por el Estado como por la comunidad". Friedman (1962) señala que esta guerra viene de antaño, los liberales se han pronunciado con argumentos económicos categóricos sobre los reales beneficios de la marihuana. Muchos intelectuales vienen promoviendo la aceleración de la despenalización de la marihuana, los psicotrópicos curativos, y el tema recreativo no se ha puesto al margen del problema. (Villa, 1986).

\section{LA ECONOMÍA SOCIAL DE MERCADO}

En el Perú, la economía social de mercado se materializa como producto de una demanda social, aprobado por el Congreso Constituyente Democrático de 1992. La libertad de competencia tiene tambien límites. El Estado ha previsto mediante la dación de normas; que, para lograr la eficiencia y la competitividad al interior del mercado, se hace necesario además frenar la concentración económica desleal buscando de este modo, el principio de igualdad y libertad para todos los integrantes de este mercado del cual hablamos. Todas las Constituciones de America del sur, ostentan aspectos económicos como son; Presupuesto, Planificacion, Propiedad Privada, Libertad de Comercio e Industria, Monopolios, Recursos naturales, y Proteccion al Consumidor principalmente. Los paises que dedican una parte especial de la Constitucion a las cuestiones económicas son; el Perú, Bolivia, Brasil, Colombia, Ecuador, y Venezuela. Por otro lado, Argentina, Chile, Uruguay, Paraguay y México, prefieren desarrollar su articulado con respecto al tratamiento económico.

\section{OBJETIVOS}

- Determinar en que nivel el aspecto legislativo de la despenalización de la marihuana (canabis sativa) para "uso terapéutico" influyen en la economía social del mercado en Tacna, año 2015.

- Determinar en que medida el aspecto judicial de la despenalización de la marihuana (canabis sativa) para "uso terapéutico" influyen en la economía social del mercado en Tacna, año 2015.

- Determinar en que nivel el aspecto político de la despenalización de la marihuana (canabis sativa) para "uso terapéutico" influyen en la economía social del mercado en Tacna, año 2015. 


\section{METODOLOGÍA}

La presente investigación fue de tipo aplicada, descriptivo-Explicativo en la modalidad Causal. Se desarrolló en la Ciudad de Tacna, en las Escuelas de Postgrado de la Universidad Privada de Tacna, Universidad Nacional Jorge Basadre Grohoman y Universidad Jose Carlos Mariategui. En esta investigación se requirió el intercambio de opiniones con profesionales del Derecho y disciplinas afines, así como de los participantes de Maestría en Ciencias Penales y de Doctorado, tanto graduados y no graduados. La muestra estuvo conformada por 91 profesionales del Derecho (17 magistrados, 41 abogados y 33 docentes del derecho). Se utilizó la encuesta y la entrevista.

\section{RESULTADOS}

El 41,2\% de los magistrados indican estar "De acuerdo", que el Estado peruano ha dado muestras de avances significativos para una despenalización de las drogas, mientras que solo un $11,8 \%$ de ellos dicen que "no estan de acuerdo", que se haya desarrollado algunos avances para despenalizar el uso de las drogas. Sin embargo, el 53,7\% de los abogados señalan estar en Desacuerdo, lo que significa que el Estado peruano aun no ha dado muestras de avances significativos para despenalizar las drogas; mientras que solo un $4,9 \%$ de ellos dicen que si hay muestras de avances para despenalizar las drogas en el Peru. El $45,5 \%$ de los docentes afirman en su mayoría que estan de acuerdo, que el Estado peruano ha dado muestras de avances y acciones para despenalizar las drogas.

Tabla 01: El Estado: provecho económico al gravar "uso terapéutico" de marihuana

\begin{tabular}{|c|cccccc|cc|}
\hline & \multicolumn{7}{|c|}{ Profesionales de Derecho } & \multirow{2}{*}{} \\
\cline { 2 - 7 } & \multicolumn{1}{|c|}{ Magistrados } & Abogados & Docentes & \multirow{2}{*}{ Total } \\
\cline { 2 - 7 } & $\mathrm{N}$ & $\%$ & $\mathrm{n}$ & $\%$ & $\mathrm{n}$ & $\%$ & $\mathrm{n}$ & $\%$ \\
\hline Muy de acuerdo & 2 & $11,8 \%$ & 0 & $0,0 \%$ & 2 & $6,1 \%$ & 4 & $4,4 \%$ \\
De acuerdo & 13 & $76,5 \%$ & 33 & $80,5 \%$ & 22 & $66,7 \%$ & 68 & $74,7 \%$ \\
No podria precisar & 2 & $11,8 \%$ & 8 & $19,5 \%$ & 9 & $27,3 \%$ & 19 & $20,9 \%$ \\
Total & 17 & $100,0 \%$ & 41 & $100,0 \%$ & 33 & $100,0 \%$ & 91 & $100,0 \%$ \\
\hline Tuente: Encuesta elaborada & & & & & & &
\end{tabular}

El 76,5\% de los magistrados, el $80,5 \%$ de los abogados y el $66,7 \%$ de los docentes indican estar "De acuerdo", en que el Estado si sacaría provecho económico al gravar impuestos por la utilización de la marihuana para "uso terapéutico (Tabla 01), pero el 58,8\% de los magistrados, el $68,3 \%$ de los abogados y el $48,5 \%$ de los docentes indican que debería aplicarse la política de prevencion y control sanitario paralelamente.

El $52,9 \%$ de los magistrados y el $65,9 \%$ de los abogados indican que existe desinterés político para explicar a la población, las ventajas terapéuticas medicinales de la marihuana. El $45,5 \%$ de los docentes indican que no pueden precisar si existe o no el desinterés político para explicar a la población de las ventajas terapéuticas de la marihuana.

Tabla 02: La despenalización de la marihuana (cannabis sativa) para "uso terapéutico" 


\begin{tabular}{|c|cccccc|cc|}
\hline \multirow{2}{*}{$\begin{array}{c}\text { ¿Está de acuerdo con la } \\
\text { despenalización de la } \\
\text { marihuana (cannabis sativa) }\end{array}$} & \multicolumn{6}{|c|}{ Profesionales de Derecho } & \multirow{2}{*}{ Total } \\
\cline { 2 - 8 } para "uso terapéutico"? & $\mathrm{n}$ & $\%$ & $\mathrm{n}$ & $\%$ & $\mathrm{n}$ & $\%$ & $\mathrm{n}$ & $\%$ \\
\hline No podria precisar. & 5 & $29,4 \%$ & 8 & $19,5 \%$ & 14 & $42,4 \%$ & 27 & $29,7 \%$ \\
De acuerdo. & 10 & $58,8 \%$ & 31 & $75,6 \%$ & 19 & $57,6 \%$ & 60 & $65,9 \%$ \\
Muy de acuerdo. & 2 & $11,8 \%$ & 2 & $4,9 \%$ & 0 & $0,0 \%$ & 4 & $4,4 \%$ \\
Total & 17 & $100,0 \%$ & 41 & $100,0 \%$ & 33 & $100,0 \%$ & 91 & $100,0 \%$ \\
\hline
\end{tabular}

Fuente: Encuesta elaborada

Respecto a la despenalización de la marihuana, el $58,8 \%$ de los magistrados, el $75,6 \%$ de los abogados y el $57,6 \%$ de los docentes manifiestan que estan "de acuerdo" con dicha despenalización para "uso terapéutico" (Tabla 02), pero el $64,7 \%$ de los magistrados, el $70,7 \%$ de los abogados y el $69,7 \%$ de los docentes estan "de acuerdo" que la regulación administrativa de la marihuana y su distribución sea en farmacias, con receta médica y previa identificación personal.

Respecto a que el Estado ha previsto que para el logro de la eficiencia y la competividad en el Mercado, es necesario poner límites a la concentración económica desleal, el 35,3\% de los magistrados, el $61,0 \%$ de los abogados y el $57,6 \%$ de los docentes señalan estar "de acuerdo" con esta afirmación. Además de lo dicho, el 76,5\% de los magistrados, el $68,3 \%$ de los abogados y el $51,5 \%$ de los docentes, manifiestan que están "de acuerdo" y que la economía social de mercado permite establacer candados jurídicos para evitar sistemas monopólicos.

El $64,7 \%$ de los magistrados, el $63,4 \%$ de los abogados y el $75,8 \%$ de los docentes están "de acuerdo" que el Estado sí interviene para asegurar una economía social de mercado óptimo, y promueve la libre competencia.

Hay una correlación positiva entre el aspecto legislativo, el aspecto judicial y el aspecto político de la despenalización de la marihuana para "uso terapéutico" y la economía social de mercado ( $\mathrm{p}$ : $0.00)$.

\section{DISCUSIÓN}

Los factores de la despenalización de la marihuana (cannabis sativa) para "uso terapéutico" influyen positivamente en la Economía Social de Mercado. El 65,9\% de los profesionales encuestados, afirman estar de acuerdo con la despenalización de la marihuana para "uso terapéutico". El artículo 25 de la Declaración Universal de Derechos Humanos establece; que toda persona tiene derecho a un nivel de vida adecuado que le asegure, así como a su familia, la salud y el bienestar, y en especial la asistencia médica, categoría importante de consenso. En el aspecto Legislativo influye positivamente en la economía social de mercado. Un $65,9 \%$ de los profesionales encuestados, señalan que sí favorecería ventajosamente la despenalización de la marihuana para "uso terapéutico" en beneficio de la sociedad. A la fecha, se ha dado un gran avance en el Congreso de la Republica al aprobar el "uso terapéutico" del cannabis. En el aspecto Judicial un $46,2 \%$ de los profesionales encuestados, afirman que la despenalización permitiría un descongestionamiento judicial con menos carga procesal, asi, el Estado se ocuparía de asuntos más relevantes que atender. Se ha comprobado que el aspecto político de la despenalización de la marihuana influye positivamente en la economía social de mercado. Un $59,3 \%$ de los profesionales encuestados, señalan que de despenalizarse la marihuana para "uso terapéutico", el Estado peruano deberá asumir una política de control sanitario y venta bajo receta médica. Finalmente, la categoría ontológica que corresponde al ser humano; es la salud pública: presupuesto necesario para lograr una calidad de vida digna. Se recomienda al Poder Ejecutivo, la reglamentación de este derecho constitucional, pues implica el bien jurídico protegido del ser 
humano, por ser tal. Lo más trascendente es atender los reclamos sociales en materia de salud. Recordemos la intervencion policial en febrero-2017, a un laboratorio clandestino de aceite de marihuana en San Miguel- Lima. Este se utilizaba para tratar convulsiones en niños. Ante la protesta popular, hoy, el Congreso de la República ha legislado en base a una necesidad social. La despenalización de la marihuana para "uso terapéutico", permitirá al Estado peruano economizar muchos recursos, esos que viene destinando para el frontal combate contra las drogas. Estos peculios podrían ser destinados para atender otras demandas sociales. La presente propuesta se dirige específicamente a la marihuana para "uso terapéutico", esto hará que dicho producto se encuentre al alcance de las regulaciones propias de una economía social de mercado, en un auténtico estado de derecho. El Ministerio de salud, el Ministerio de Agricultura, y el Ministerio del Interior en su labor de control, deberán hacer cumplir las disposiciones del gobierno, además de los estudios médicos, y las Instituciones universitarias, para que dediquen un esfuerzo en el estudio científico de la planta, en la medida que fomente solo para "uso terapéutico". Es una demanda social largamente esperada. Tendremos que aprender a coexistir con esta planta, ya lo hemos hecho con el alcohol o tabaco. América del Sur es el paradigma de ello; caso Uruguay, Chile, Colombia, y otros. Propongo el modelo canadiense para el Perú. Por las consideraciones expuestas, recomiendo al Poder Ejecutivo, un modelo de reglamentación penal más humano y viable a la realidad actual de nuestro país, que garantice el alcance a todos los peruanos, de la planta del cáñamo hembra denominado; marihuana (cannabis sativa), para "uso terapéutico". Ello, justificado en el principio y fundamento metafísico de los Derechos Humanos. Kant proclamaba: "El hombre no puede ser tratado por ningún otro, ni siquiera por el mismo; como un medio, sino siempre como un fin, y que justamente en ello radica su dignidad".

\section{REFERENCIAS BIBLIOGRÁFICAS}

Baratta, A. (1993). Introduccion a una sociología de la droga, en Revista Juridica, N 27. Ecuador. Editorial. Universidad Catolica de Santiago de Guayaquil.

Bouquet, J. (1950). La marihuana Toro de cannabis. Narcotráfico. En https:/www.scribd.com/document/331158612/cannabis

Blickman, T. y Jelsma, M. (2012). La Reforma de las Politicas de Drogas.Holanda. Editorial Nueva Sociedad.

Fernandez, C. (2013). La evidencia en contra de la legalización de la marihuana. Mexico. Ediciones UNAM.

Fetterman, D. (1970). Avances en farmacología y quimioterapia. Gran Bretaña. Garattini, Goldin, Hawking, Irwin, Kopin

Friedman, M. (1962). Libertad de elegir. Hacia un nuevo liberalismo económico. Barcelona. Ediciones Grijalbo.

Garat, G. (2011). Marihuana y otras hierbas. Uruguay. Editorial Debate.

Kramer J. y Cameron D. (1975). Manual sobre la dependencia de drogas. Ginebra. OMS.

Peyraube, R. y Bouso, J. (2015). ¿Marihuana como medicina? Mexico.UNAM.

Mechoulam, R. (2005). Delta-9- Tetrahidrocannabinol. Jerusalen. Editorial Alianza.

Turk, W. (1970). Metodo quimico simple para identificar la marihuana. ONU. Stanton.

Villa, S. (1986). Aceleremos la legalización de todas las drogas. España. Editorial Edersa. 